\title{
Syphilis in London circa 2004: new challenges from an old disease
}

\author{
A Cross, S Luck, R Patey, M Sharland, P Rice, R Chakraborty
}

Arch Dis Child 2005;90:1045-1046. doi: 10.1136/adc.2004.069377

ra recent decades, cases of newly diagnosed syphilis were rare in the United Kingdom. Since 1996, however, the number of cases of primary and secondary syphilis in women has increased threefold. ${ }^{12}$ This resurgence has been associated with a number of localised outbreaks, which have affected women of childbearing age and has been linked to the birth of at least one infant with documented congenital syphilis. ${ }^{3}$ Antenatal screening facilitates treatment of infectious syphilis during pregnancy and offsets vertical transmission and congenital infection. ${ }^{4}$ Here we report on the inadequate investigation, treatment, and follow up of pregnant women with positive antenatal syphilis serology and their infants, attending clinics at a tertiary hospital in southwest London. If similar problems are encountered at hospitals nationally, cases of congenital syphilis may go unrecognised within the UK.

Given the previously low rates of congenital syphilis, clinicians may be currently relatively unfamiliar with the diagnosis and management of a potentially fatal but preventable condition in infants. Guidelines exist in both the UK and North America, ${ }^{5}{ }^{6}$ but there are concerns that these are not being uniformly followed. In view of the increasing rate of syphilis nationally we carried out a clinical audit of the completeness of investigation, treatment, and follow up of pregnant women with positive antenatal syphilis serology and their infants at a tertiary hospital in south London.

\section{METHODS}

A retrospective audit was carried out at St George's Hospital NHS Trust between June 2000 and August 2003 of all pregnant women with positive first line syphilis serology (VDRL, RPR, or TPPA) at booking. Standard data were collected from maternal antenatal and genitourinary medicine case notes, and from results of additional serological testing for syphilis. Where possible data were collected from all three sources to obtain a full record of all serological results, referrals made, treatment given, and further antenatal follow up.

The case notes of infants born to seropositive women were also reviewed where available and results of serological tests noted. Evidence of serological testing of exposed infants was reviewed again in March 2004.

\section{RESULTS}

Abnormal serology for syphilis from initial screening was noted in 72/12 600 pregnant women booking in the antenatal clinic at St George's hospital over 38 months (0.6\%). Further serological data were available for 70 women. Nine were considered to have false positive reactivities since all further serology was unreactive. Biological false positive reactions (consistently reactive non-treponemal titres in the presence of unreactive repeat specific treponemal serology) were noted in 17. IgM to syphilis (which in the absence of a history of recent treatment suggests active disease) was identified in four patients. The remaining women had results which would warrant further evaluation and follow up to ascertain whether there was active, or previously treated syphilis (see fig 1). A total of $8 / 70$ women (11\%) with positive nontreponemal titres by initial screening, however, had incomplete serological analysis.

A total of 24/70 women (34\%) attended the genitourinary clinic for further management. Because of the nature of data collection it is unknown how many women were referred but failed to attend. Four were false positive or biologically false positive. Eleven received treatment for syphilis. Of these, two received an incomplete course of treatment (as a result of allergy to penicillin and failure to follow up, respectively). A further six of 11 treated women had no documented decrease in non-treponemal antibody titres. In total $8 / 11(72 \%)$ had insufficient treatment and/or follow up to offset vertical transmission. Four women had previous treatment documented and were negative on non-treponemal testing. The remainder were not treated or had incomplete follow up.

Sixty two per cent $(26 / 42)$ of women who were considered to be at intermediate risk of transmitting infectious syphilis to their newborn infants received no further follow up or serology. Our definition of intermediate risk was women who on initial screening were not known to be "high risk" as defined by positive IgM, but could not be put in a low risk group (that is, previously treated syphilis, false positive screening tests) without further assessment. In addition, only $40 \%(13 / 42)$ of infants born to women in this group received serological evaluation or follow up for syphilis.

Sixty one infants were born at St George's Hospital to 60 women (four women delivered in other hospitals, two pregnancies were terminated, three were lost to follow up). In addition there was one intrauterine death, unrelated to syphilis at postmortem examination. Sixteen of 46 (35\%) infants at risk of congenital infection had serological testing. Babies were considered to be at risk if they were born to mothers who were either IgM positive or who had inadequate antenatal evaluation or treatment to exclude active infection. All were TPPA positive at birth but by 6 months of age only two remained positive. Six babies did not complete follow up-that is, when last evaluated they continued to be TPPA positive and follow up was not completed to one year as recommended. ${ }^{5}$ No infant was born with clinical features of congenital syphilis and no babies were FTA-ABS IgM positive during the study period.

\section{DISCUSSION}

An inherent problem in a retrospective audit of this kind is the collection of complete data for all patients, especially when records are held in different departments within the hospital.

The results of this study support our concerns, however, that there is inadequate investigation, treatment, and follow up of pregnant women with positive antenatal syphilis serology and their infants. In a climate of increasing syphilis infection in women of childbearing age this may lead to 

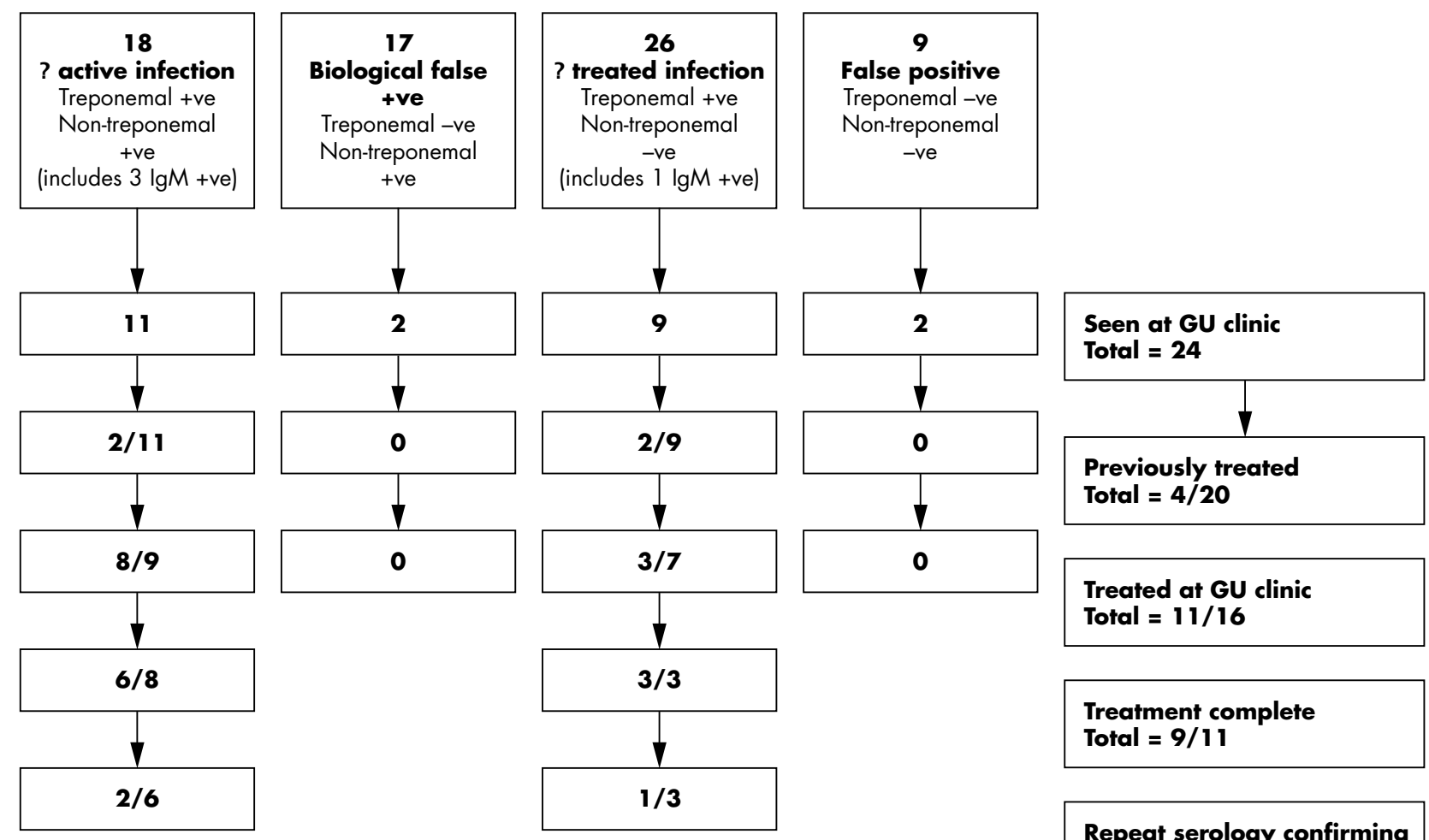

Treatment complete

Total $=9 / 11$

Repeat serology confirming
treatment complete
= patients sufficiently
treated to ensure no risk
of congenital infection
Total $=3 / 9$

Figure 1 Documented outcome for 70 pregnant women with positive syphilis serology within St George's Hospital.

unrecognised cases of congenital syphilis in the United Kingdom. Difficulties encountered during this study in accessing full records for women and tracking their follow up following positive antenatal screening tests raised concerns that the management of mothers and babies may be hindered by lack of centralised coordination, resulting in existing guidelines ${ }^{5}$ not being followed. Further discussion locally has confirmed these concerns and steps have now been taken to ensure that antenatal screening results are reviewed by a multidisciplinary team with experience in the management of syphilis. This enables a full evaluation of the mother to be carried out antenatally and a plan made for further evaluation, treatment, and follow up of the neonate. We hope that when a re-audit is carried out locally we can show an improvement in our management of antenatal syphilis. However, we continue to have concerns about how the problem is being managed nationally.

\section{ACKNOWLEDGEMENTS}

Dr P Hay provided serological data for the study.

\section{Authors' affiliations}

A Cross, S Luck, R Patey, M Sharland, P Rice, R Chakraborty,

Department of Child Health, St George's Hospital, London, UK
Competing interests: none

Correspondence to: Dr R Chakraborty, Department of Child Health, St George's Hospital, 5th Floor, Lanesborough Wing, Blackshaw Road, Tooting, London SW17 0QT, UK; ranachakraborty@hotmail.com

Accepted 30 May 2005

\section{REFERENCES}

1 Hurtig A-K, Nicoll A, Carne C, et al. Syphilis in pregnant women and their children in the United Kingdom: results from national clinician reporting surveys 1994-7. BMJ 1998;317:1617-19.

2 Communicable Disease Surveillance Centre. Summary tables of selected conditions by sex: England, Wales and Northern Ireland* 1996-2002, Communicable Disease Surveillance Centre (HIV and STI Section).

3 Anon. Evidence for increased transmission of syphilis among homosexual men and heterosexual men and women in Europe. Eurosurveillance Weekly Archives 2000;4(50).

4 Beilinson P. Epidemic of congenital syphilis-Baltimore, 1996-1997. CDC MMWR 1998;47:904-7

5 Association for Genitourinary Medicine (AGUM), Medical Society for the Study of Venereal Disease (MSSVD). 2002 national guidelines on the management of early syphilis. London: Association for Genitourinary Medicine (AGUM), Medical Society for the Study of Venereal Disease (MSSVD), 2002.

6 Centers for Disease Control and Prevention. Congenital syphilis. Sexually transmitted diseases treatment guidelines. MMWR Recomm Rep 2002;51(RR-6):26-8. 